\title{
PERSONALITY AND BEHAVIORAL PATTERNS OF PATIENTS WITH VARIOUS HORMONALLY ACTIVE PITUITARY ADENOMAS
}

\begin{abstract}
A.E. Bobrov¹, E.G. Starostina², M.M. Alexandrova1, D.R. Almaev¹, Z.E. Belaya³ , M.G. Pavlova ${ }^{4}$, I.V. Komerdus², G.A. Melnichenko ${ }^{5}$

'V.Serbsky National Medical Research Centre for Psychiatry and Narcology, Consultative and Distance Psychiatry, Moscow, Russia. ${ }^{2}$ Moscow Regional Clinical and Research Institute, Clinical Endocrinology, Moscow, Russia.

${ }^{3}$ National Medical Research Center for Endocrinology, Department of Neuroendocrinology and Bone Disease, Moscow, Russia.

4I.M. Sechenov First Moscow State Medical University (Sechenov University), Department of Endocrinology № 1, Moscow, Russia.

${ }^{5}$ National Medical Research Center for Endocrinology, Institute of Clinical Endocrinology, Moscow, Russia.
\end{abstract}

\section{Objectives:}

Mental disorders associated with various pituitary adenomas are clinically different [1].

\section{Background and Aims:}

The aim of the study was to identify patterns of personality and behavior underlying these differences.

\section{Materials and Methods:}

This cross-sectional cohort study included three patient groups: 90 with somatotropinomas (ST, aged $55.2+12.7$ years, M/F 14/76), 80 with corticotropinomas (CT, aged $40.3+12.5$ years, $\mathrm{M} / \mathrm{F} 8 / 72$ ), and 55 with prolactinomas ( $\mathrm{P}$, aged $33.3+9.3$ years, $\mathrm{M} / \mathrm{F}$ 0/55). All of them were interviewed by psychiatrists and tested with MMPI. These groups were further divided into the active phase (AP) and treatment-compensated phase (CP) subgroups, depending on their hormonal levels. The data were analyzed with ANOVA at $p<0.05$

\section{Results and Conclusions:}

There were significant differences in average MMPI scale scores depending on the type of tumors and their secretory activity. The ST AP subgroup was characterized by higher conformity (scale K) and internal behavioral standards (scale L), while ST CP patients had higher social introversion ( $\mathrm{Si}$ scale). CT AP patients demonstrated a prominent decrease in gender differentiation (Mf scale), while CT CP subgroup had the lowest anxiety level (Pt scale). P AP was characterized by increased impulsivity ( $\mathrm{Pd}$ scale), while $\mathrm{P}$ CP subgroup had the highest anxiety (Pt scale) and worst adjustment scores. Behavioral patterns of patients with various hormonally active pituitary adenomas have significant differences, which are apparently associated with the secretory activity of the tumors.
Suppression of pituitary hypersecretion can lead to psychologically important changes in behavior, which should be considered in the rehabilitation of such patients.

\section{Table 1. MMPI Scores of Patients with Various Pituitary} Adenomas: Active Phase

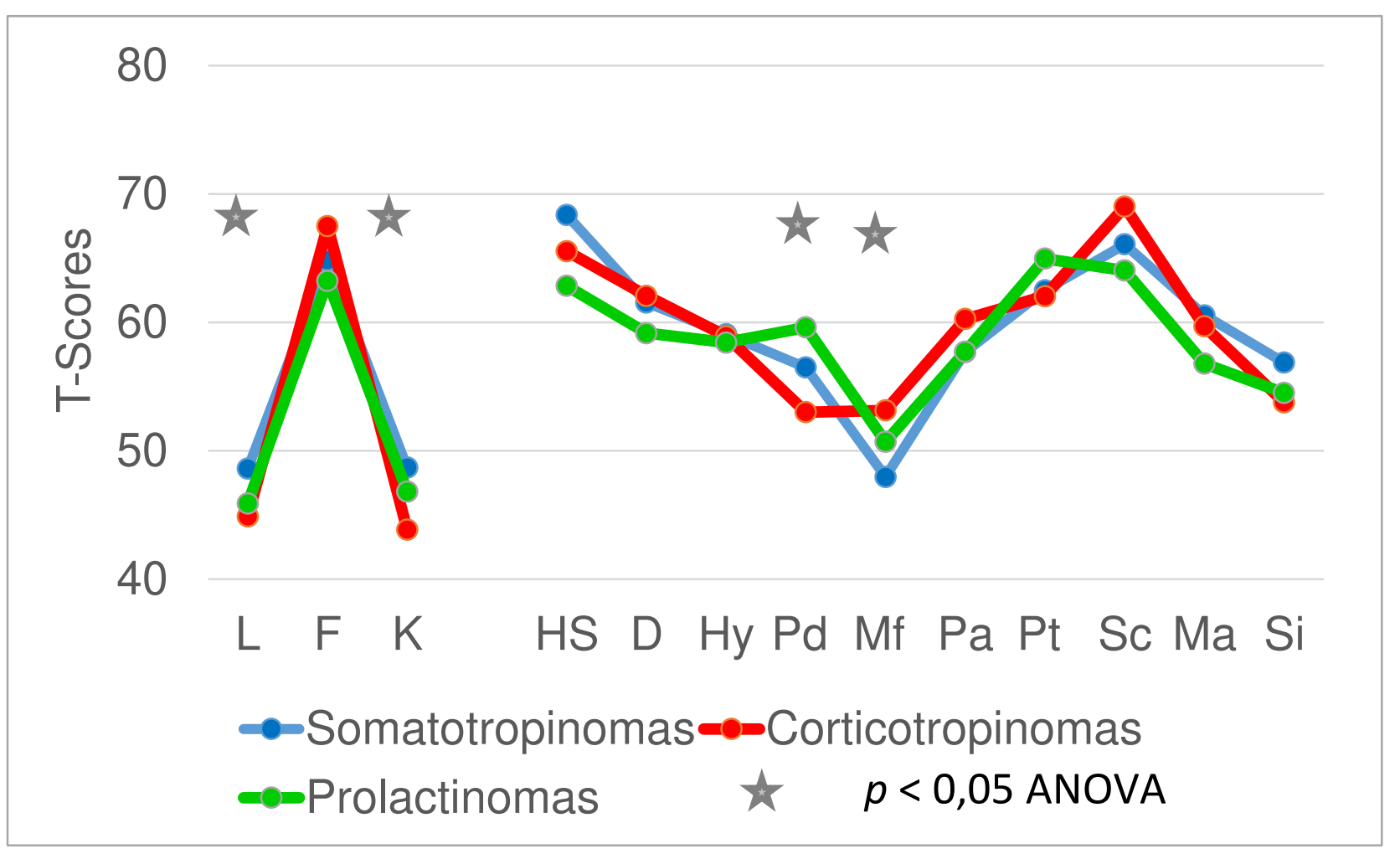

Table 2. MMPI Scores of Patients with Various Pituitary Adenomas:

Treatment-Compensated Phase

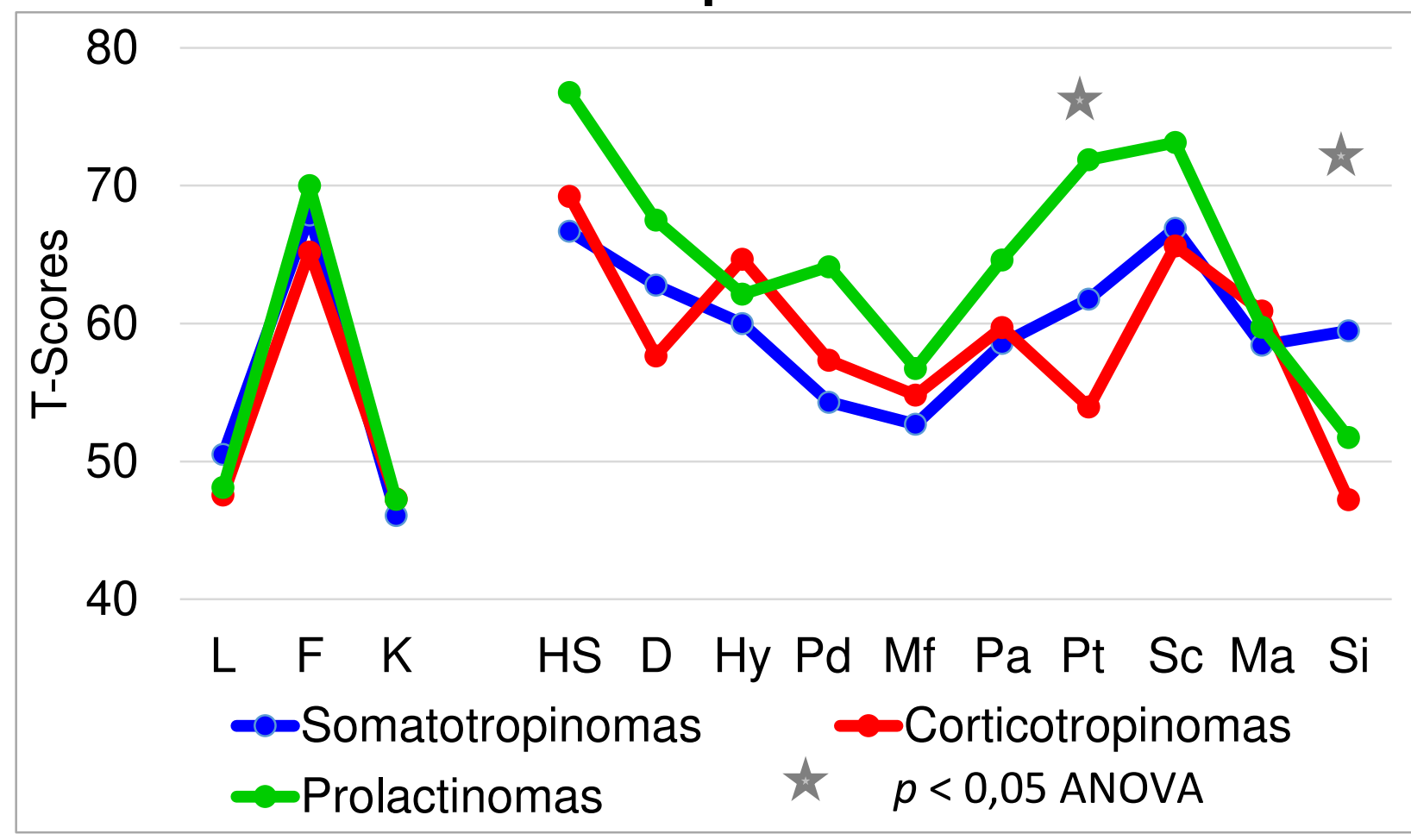

Chart. Average MMPI Scores for Treatment-Compensated and Active Phases in Patients With Various Pituitary Adenomas ( $p$ - One-Way ANOVA)

\begin{tabular}{|c|c|c|c|c|c|c|c|c|c|c|c|c|c|c|c|}
\hline $\begin{array}{c}\text { Adenomas } \\
\text { Types }\end{array}$ & $\mathbf{N}$ & Phase & $\mathbf{L}$ & $F$ & $\mathbf{K}$ & Hs & D & Hy & Pd & Mf & $\mathrm{Pa}$ & Pt & Sc & Ma & Si \\
\hline \multirow{3}{*}{$\begin{array}{l}\text { Somato- } \\
\text { tropinomas }\end{array}$} & 23 & $\begin{array}{c}\text { Compen- } \\
\text { sated }\end{array}$ & 50,5 & 67,9 & 46,1 & 66,7 & 62,8 & 60,0 & 54,3 & 52,7 & 58,6 & 61,8 & 66,9 & 58,4 & 59,5 \\
\hline & 67 & Active & 48,6 & 64,8 & 48,7 & 68,4 & 61,5 & 59,1 & 56,5 & 48,0 & 57,7 & 62,5 & 66,1 & 60,6 & 56,9 \\
\hline & & ANOVA p & 0,38 & 0,31 & 0,17 & 0,61 & 0,68 & 0,72 & 0,43 & 0,05 & 0,78 & 0,82 & 0,80 & 0,40 & 0,30 \\
\hline \multirow{3}{*}{$\begin{array}{c}\text { Cortico- } \\
\text { tropinomas }\end{array}$} & 21 & $\begin{array}{l}\text { Compen- } \\
\text { sated }\end{array}$ & 47,6 & 65,2 & 47,3 & 69,2 & 57,7 & 64,7 & 57,3 & 54,8 & 59,7 & 54,0 & 65,6 & 60,9 & 47,2 \\
\hline & 59 & Active & 44,9 & 67,5 & 43,9 & 65,5 & 62,1 & 58,9 & 53,0 & 53,2 & 60,3 & 62,1 & 69,0 & 59,7 & 53,8 \\
\hline & & ANOVA p & 0,29 & 0,44 & 0,20 & 0,25 & 0,15 & 0,03 & 0,20 & 0,51 & 0,88 & 0,02 & 0,40 & 0,69 & 0,02 \\
\hline \multirow{3}{*}{$\begin{array}{l}\text { Prolacti- } \\
\text { nomas }\end{array}$} & 8 & $\begin{array}{l}\text { Compen- } \\
\text { sated }\end{array}$ & 48,1 & 70,0 & 47,3 & 76,8 & 67,5 & 62,1 & 64,1 & 56,8 & 64,6 & 71,9 & 73,1 & 59,8 & 51,8 \\
\hline & 47 & Active & 45,9 & 63,2 & 46,8 & 62,9 & 59,2 & 58,4 & 59,6 & 50,7 & 57,7 & 65,0 & 64,0 & 56,8 & 54,5 \\
\hline & & ANOVA p & 0,46 & 0,17 & 0,89 & 0,02 & 0,11 & 0,46 & 0,38 & 0,14 & 0,16 & 0,31 & 0,17 & 0,48 & 0,49 \\
\hline
\end{tabular}

